\title{
Wave Propagation Simulation of Complex Multi-Material Problems with Fast Low-Order Unstructured Finite-Element Meshing and Analysis
}

\author{
Kohei Fujita \\ Earthquake Research Institute \& \\ Department of Civil Engineering, \\ The University of Tokyo \\ Advanced Institute for \\ Computational Science, RIKEN \\ fujita@eri.u-tokyo.ac.jp \\ Masashi Horikoshi \\ Software and Solutions Group, \\ Intel K.K. \\ Masashi.Horikoshi@intel.com
}

\author{
Keisuke Katsushima \\ Earthquake Research Institute \& \\ Department of Civil Engineering, \\ The University of Tokyo \\ keisuke-k@eri.u-tokyo.ac.jp
}

\author{
Tsuyoshi Ichimura \\ Earthquake Research Institute \& \\ Department of Civil Engineering, \\ The University of Tokyo \\ Advanced Institute for \\ Computational Science, RIKEN \\ ichimura@eri.u-tokyo.ac.jp \\ Muneo Hori \\ Earthquake Research Institute \& \\ Department of Civil Engineering, \\ The University of Tokyo \\ Advanced Institute for \\ Computational Science, RIKEN \\ hori@eri.u-tokyo.ac.jp
}

\begin{abstract}
Many wave-propagation analyses with varying geometries and material properties are expected to be useful for model optimization. Low-order unstructured finite-element methods are suitable for such analyses, as they are capable of modeling multi-material problems with complex geometries; however, the meshing and analysis cost is large. Therefore, in this paper, we developed a fast mesh-generator and analysis method. The robust mesh generator was 17.4-fold faster than a conventional mesh generator, and the predictor algorithm for dynamic implicit finite-element solvers showed a 1.69-fold increase in speed relative to conventional solvers and a $91.3 \%$ size-up efficiency on the full Oakforest-PACS system. We demonstrated the usability of the developed meshing and
\end{abstract}

Permission to make digital or hard copies of all or part of this work for personal or classroom use is granted without fee provided that copies are not made or distributed for profit or commercial advantage and that copies bear this notice and the full citation on the first page. Copyrights for components of this work owned by others than ACM must be honored. Abstracting with credit is permitted. To copy otherwise, or republish, to post on servers or to redistribute to lists, requires prior specific permission and/or a fee. Request permissions from permissions@acm.org.

HPC Asia 2018, January 28-31, 2018, Chiyoda, Tokyo, Japan

(C) 2018 Association for Computing Machinery.

ACM ISBN 978-1-4503-5372-4/18/01 ...\$15.00

https://doi.org/10.1145/3149457.3149474 analysis methods via a wave-propagation simulation on a 1.9 billion unstructured tetrahedral-element model using half of the K computer system (41,472 compute nodes).

\section{KEYWORDS}

finite-element method, tetrahedral mesh generation, adaptive multistep method, wave-propagation

\section{ACM Reference Format:}

Kohei Fujita, Keisuke Katsushima, Tsuyoshi Ichimura, Masashi Horikoshi, Kengo Nakajima, Muneo Hori, and Lalith Maddegedara. 2018. Wave Propagation Simulation of Complex Multi-Material Problems with Fast Low-Order Unstructured Finite-Element Meshing and Analysis. In Proceedings of International Conference on High Performance Computing in Asia-Pacific Region, Chiyoda, Tokyo, Japan, January 28-31, 2018 (HPC Asia 2018), 12 pages. https://doi.org/10.1145/3149457.3149474

\section{INTRODUCTION}

Many cases of wave-propagation analyses are used for engineering and medical purposes for model optimization (e.g., 
nondestructive inspection of structures, subsurface tomography for resource development, and extraction of medical images). In these problems, the geometry and nonuniform material properties of the target domain greatly affect wave-propagation characteristics. Three-dimensional loworder unstructured finite-element methods are suitable for multi-material problems with complex geometries; however, their meshing and analysis costs become large. Therefore, simplified analysis methods are often used for model optimization of wave-propagation problems, with few research projects using three-dimensional unstructured finite-element methods. As an example of analysis using three-dimensional finite-element methods, [1] repeats mesh generation and wavepropagation analysis of underground crust structure to improve the underground crust model. This is enabled by accelerating the entire forward simulation, including the meshing and wave-propagation analyses, using a fast and robust mesh generator and analysis methods specialized for the layered crust-structure problem [2, 3]. By developing a fast and robust mesh generator and an analysis method for general multi-material and complex geometry problems, we expect improvements in model optimization for a wide range of problems. Currently, meshing of multi-material problems with complex geometries is challenging from the viewpoints of robustness and mesh-generation time, and the speed associated with finite-element wave-propagation analysis is not sufficient for many case analyses. Therefore, in this study, we developed a fast low-order unstructured finite-element meshing and analysis method capable of computing wave propagation problems in complex multi-material domains.

The remainder of the paper is organized as follows. In Section 2, we explain the target problem. In Section 3, we explain the fast and robust mesh-generator method. In Section 4, we explain the adaptive multistep predictor method developed to accelerate finite-element analyses. In Section 5, we show an example of wave-propagation analysis of an underground structure in Tokyo station. Section 6 summarizes the paper.

\section{TARGET PROBLEM}

We target many linear wave-propagation analyses in complexshaped domains with highly nonuniform material properties. Therefore, we used a three-dimensional dynamic finiteelement method with low-order unstructured elements, because the method analytically satisfies the traction-free boundary condition and is suitable for modeling complex geometry and material heterogeneity with low computation costs. The target equation becomes as below:

$$
\begin{array}{r}
\left(\frac{4}{d t^{2}} \mathbf{M}+\frac{2}{d t} \mathbf{C}+\mathbf{K}\right) \mathbf{u}^{n}= \\
\mathbf{f}^{n}+\mathbf{C v}^{n-1}+\mathbf{M}\left(\mathbf{a}^{n-1}+\frac{4}{d t} \mathbf{v}^{n-1}\right),
\end{array}
$$

where

$$
\left\{\begin{array}{l}
\mathbf{v}^{n}=-\mathbf{v}^{n-1}+\frac{2}{d t}\left(\mathbf{u}^{n}-\mathbf{u}^{n-1}\right), \\
\mathbf{a}^{n}=-\mathbf{a}^{n-1}-\frac{4}{d t} \mathbf{v}^{n-1}+\frac{4}{d t^{2}}\left(\mathbf{u}^{n}-\mathbf{u}^{n-1}\right) .
\end{array}\right.
$$

Here $\mathbf{u}, \mathbf{v}, \mathbf{a}$, and $\mathbf{f}$ represent displacement, velocity, acceleration, and external-force vectors, respectively. $\mathbf{M}, \mathbf{C}, \mathbf{K}, d t$, and $n$ represent the consistent-mass, damping, and stiffness matrices, time increment, and time step, respectively. We use Rayleigh damping for $\mathbf{C}$, where the element-damping matrix, $\mathbf{C}_{e}$, is calculated using the element-consistent-mass matrix $\mathbf{M}_{e}$ and the element-stiffness matrix $\mathbf{K}_{e}$ as $\mathbf{C}_{e}=\alpha \mathbf{M}_{e}+\beta \mathbf{K}_{e}$. Here $\alpha$ and $\beta$ are obtained by solving the following leastsquares equation:

$$
\operatorname{minimize}\left[\int_{f_{\min }}^{f_{\max }}\left(h-\frac{1}{2}\left(\frac{\alpha}{2 \pi f}+2 \pi f \beta\right)\right)^{2} \mathrm{~d} f\right] .
$$

Here $f_{\max }, f_{\min }$, and $h$ represent the maximum and minimum target frequencies and the damping ratio, respectively. Note that small elements can be generated in parts of the domain when meshing complex geometries using solid elements. Under such conditions, a very small time increment is required to satisfy the Courant condition; therefore, explicit time-integration methods (e.g., the central-difference method) lead to huge computation costs. Consequently, we used the Newmark- $\beta$ method $(\beta=1 / 4, \delta=1 / 2)$ for time integration. Because the numerical solution depends upon $d t$ and spatial discretization, we assigned the problem setting and discretization size to ensure numerical convergence of the solution. In summary, time-history response, $\mathbf{u}^{n}$, is computed by repeating the following steps:

(1) Read boundary conditions

(2) Obtain $\mathbf{u}^{n}$ by solving Eq. (1).

(3) Update Eq. (2) using $\mathbf{u}^{n}$.

In practical problems, a conforming mesh with a large number of elements is required for analysis, along with remeshing for model optimization. In addition, solving Eq. (1) requires indirect memory access and large memory transfer, making it difficult to attain high performance and scalability using recent large-scale computers. In Section 3, we show the mesh-generation method for solving the above finite-element wave-propagation problem. In Section 4, we show the analysis method used to accelerate the finite-element solver on massively parallel computers.

\section{FAST AND ROBUST MESHING METHOD FOR COMPLEX MULTI-MATERIAL PROBLEMS}

In typical wave-propagation problems, geometric features and material heterogeneity that are long relative to target wavelengths affect the solutions; therefore, a large-scale mesh is required to resolve these problem characteristics. However, robust meshing of large-scale problems with multiple materials and complex geometries remains challenging, and mesh generation remains a bottleneck throughout the entire wavepropagation analysis process. Therefore we developed a parallel and robust tetrahedral finite-element mesh-generation method capable of modeling multi-material problems with complex shapes [4]. In this method, the conformity and quality (i.e., aspect ratios of elements) of the generated mesh are robustly ensured by allowing small approximations in 
geometries shorter than the target wavelengths. Although the method is suited for parallel processing, it is slow when considering re-meshing during analysis or targeting huge problems with billions of elements. Therefore, we enhanced this method to develop a fast and robust meshing method. First, we summarize the previous study [4], followed by the explanation of the developed meshing method.

\subsection{Overview of the previous meshing method}

Using closed triangular patches for each material as inputs, this method generates a conforming finite-element mesh with linear tetrahedral elements. First, the target domain is decomposed into multi-scale cubes using an orthogonal octree structure, and each cube is decomposed into tetrahedral elements. We refer to the former process as "cube generation" and the latter process as "tetrahedral mesh generation."

3.1.1 Cube generation. Based on an orthogonal octree structure, background cubes are generated in the target domain. This process comprises three steps.

Top-down cube generation. First, a cube that covers the target domain, including all input triangle patches, is generated (Fig. 1a). Cubes with input triangle patches are recursively decomposed into eight cubes using an orthogonal octree structure (Fig. 1b). The recursive decomposition is terminated when the leaf cube reaches a prescribed cube size (referred to as "resolution").

Node coloring. All of the vertices of the generated cubes are colored according to the material-identification number (Fig. 1c). Here only vertices near material boundaries are colored by an inside/outside judgment algorithm, with a filling algorithm used to color the interior nodes.

Cube refinement. To attain numerical accuracy of the wavepropagation solution, we further decompose cubes recursively until the leaf-cube size reaches a prescribed size defined for each material. Cubes are recursively decomposed until the size of neighboring cubes is within one level of difference (Fig. 1d). This process avoids rapid changes in element size, which circumvents the generation of squashed elements that often leads to noise in numerical solutions and degrades the convergence characteristics of the finite-element iterative solvers.

3.1.2 Tetrahedral mesh generation. We then tetrahedralize each cube based on the orthogonal octree structure. Here we use different meshing procedures depending upon the existence of material boundaries inside the target cube.

Cubes with material boundaries. In this case, some of the eight vertices of the cube have different colors. Based on the colors of the eight vertices (Fig. 2a), we first generate triangulated material-boundary surfaces inside each cube (Fig. 2b) using the multi-material marching-cubes method [5], which generates boundary triangular patches using the material coloring at the eight vertices. Contrary to the original marching-cubes method, which was limited to two materials,

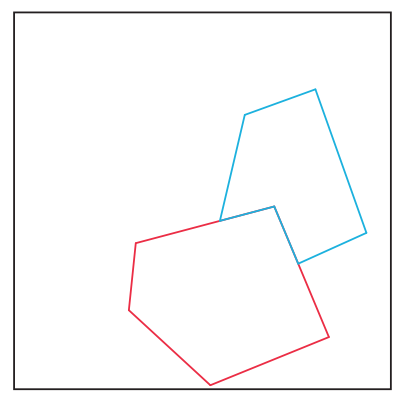

(a)

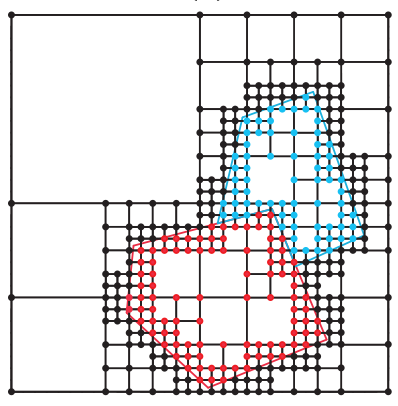

(c)

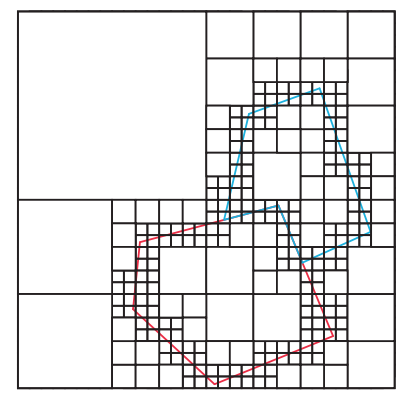

(b)

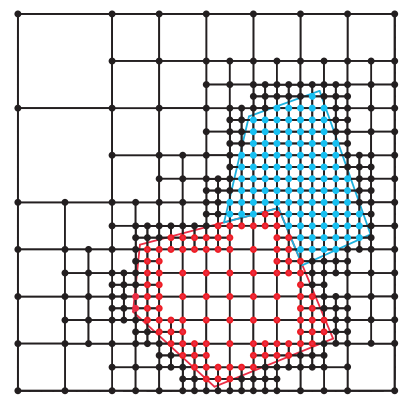

(d)
Figure 1: Cube-generation procedures in two dimensions. Actual cube generation is undertaken using an orthogonal octree structure.

the multi-material marching-cubes method can robustly generate boundary surface patches for three or more materials. In the multi-material marching-cubes method, new nodes can only be generated at the center of the cube edges, at the center of cube faces, or at the center of the cube. This enables robust meshing of multi-material problems in exchange for geometrical approximation of half of the leaf-cube size. Next, the six faces of each cube are decomposed into triangle patches using Delaunay triangulation (Fig. 2c). To ensure the uniqueness of triangulation between neighboring cubes, decomposition patterns are predetermined for special inputs with ambiguity. This ensures the conformity of the mesh across cube faces without synchronization among cubes. Each material region is tetrahedralized according to constrained Delaunay tetrahedralization [6] using the material boundary and cube-boundary patches (Fig. 2d). Because these patches conform across cubes, the generated tetrahedral elements also conform automatically.

Cubes without material boundaries. In this case, all of the vertices have the same color; however, the existence of nodes on the cube edges and faces depends on the octree structure. Therefore, we first triangulate the six faces of the cube using Delaunay triangulation. Here there can be 12 edge-centered nodes and six face-centered nodes in each cube (Fig. 3a and $3 \mathrm{~b}$ ). The cube is then decomposed using constrained Delaunay tetrahedralization while maintaining the surface patches (Fig. 3c). 


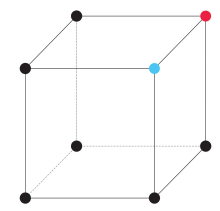

(a)

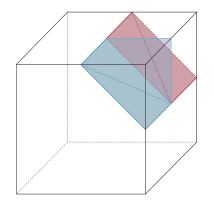

(b)

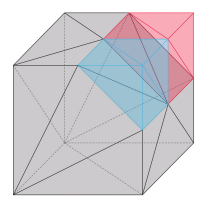

(c)

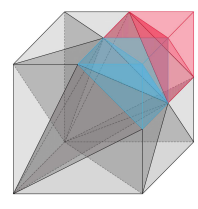

(d)
Figure 2: Boundary cube decomposition used used in the previous method

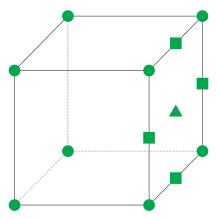

(a)

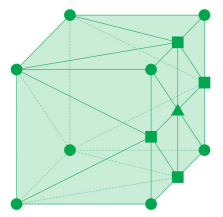

(b)

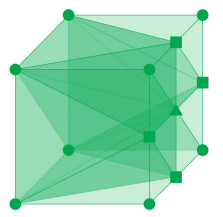

(c)
Figure 3: Interior cube decomposition used in the previous method

In summary, by constraining the positions of the generated nodes, the previously described meshing method [4] is capable of robustly meshing multi-material problems with complex geometries in parallel. Acceleration of this method is expected to enable fast and robust meshing of complex multi-material problems.

\subsection{The meshing method presented in this study}

In the previous study, the node-coloring procedure and tetrahedral mesh-generation procedure dominated the total meshgeneration time. Here we accelerated the meshing method by improving the algorithms associated with both procedures.

3.2.1 Node coloring. In the previous method, coloring of nodes on material boundaries was based on the solidangle algorithm (Fig. 4), with the solid angle representing the projected area of the input triangle patch on a unit sphere centered on the target node. Because the input triangle patches have front/reverse information, we can calculate the projected area with a signed value. The node is judged inside of the patch if the area becomes $4 \pi$, and judged outside of the patch if the area becomes 0 .

The solid angle-algorithm is robust, but its cost is proportional to the number of triangles in each patch. Instead, we used the crossing-number algorithm (Fig. 5), which counts the number of intersections between the triangle patches and a ray emitted from the target node. The node is judged inside of the patch if the number of intersections is odd. By using this method, we can eliminate the need to check most of the triangles, which significantly reduces coloring cost. Because the crossing-number algorithm is sensitive to triangles being close to the node or when the triangle becomes perpendicular to the ray, we used the solid-angle algorithm in these cases to avoid numerical errors.

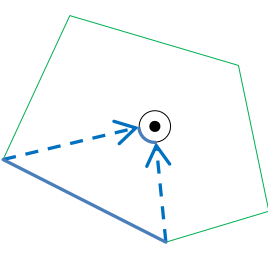

(a) Inside

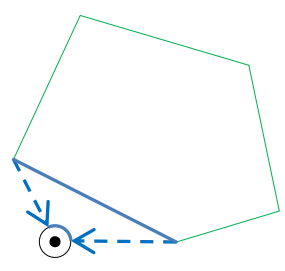

(b) Outside
Figure 4: Solid-angle algorithm for node coloring used in the previous method

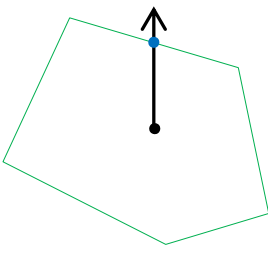

(a) Inside

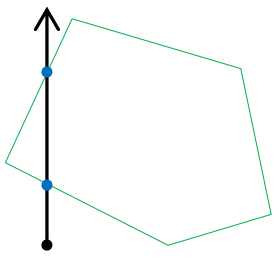

(b) Outside
Figure 5: Crossing-number algorithm for node coloring used in the meshing method presented in this study

3.2.2 Tetrahedral mesh generation. Because the tetrahedralization of a cube is unique for each node-coloring or node-existence pattern, we used a lookup table of precomputed tetrahedralization patterns to increase the speed of the tetrahedral mesh-generation process.

Cubes with material boundaries. Because the tetrahedralization pattern of a cube is unique for a given input vertex coloring pattern, we skipped all of the calculations involved in the multi-material marching-cubes method, Delaunay triangulation, and the tetrahedralization methods by using a precomputed lookup table (Fig. 6). Because the simplest lookup table will contain $8^{8}=16,777,216$ entries, this size will be too large when considering memory capacity. Therefore, we eliminated duplicate patterns, resulting in a smaller lookup table containing 20,048 entries (Table 1). The key to using this table efficiently is to make fast referencing to the entries. Because most cubes exhibit only two or three different material colors, we retained all of the duplicate cases to reduce conditional checks and to promote fast referencing. For cases with additional materials, we used a specialized reference method for fast referencing, as summarized in the Appendix. The maximum aspect ratio of the tetrahedral elements in the lookup table is 11.5, which guarantees the high quality of the generated mesh. Here the aspect ratio, $a$, represents an indicator of element quality and is defined as

$$
a=\sqrt{\frac{2}{3}} \frac{l_{\max }}{h_{\min }},
$$

where $l_{\max }$ represents the length of the longest edge of a tetrahedron and $h_{\text {min }}$ represents the shortest length of the line perpendicular from the vertex to face of a tetrahedron. 


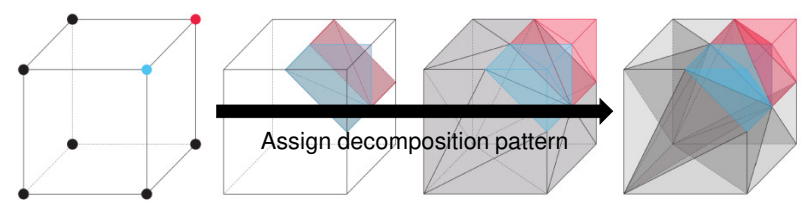

Figure 6: Boundary cube decomposition used in the method presented in this study

Table 1: Boundary cube-decomposition lookup table

\begin{tabular}{lccccccc}
\hline \# of materials & 2 & 3 & 4 & 5 & 6 & 7 & 8 \\
\hline \# of entities & 256 & 6561 & 9016 & 3710 & 476 & 28 & 1 \\
\hline
\end{tabular}
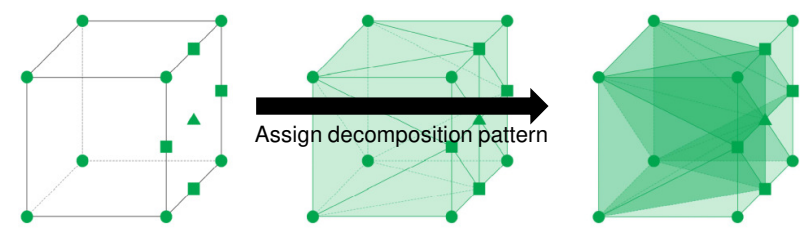

Figure 7: Interior cube decomposition used in the method presented in this study

Table 2: Interior cube-decomposition lookup table

\begin{tabular}{lccccccc}
\hline \# of face-centered nodes & 0 & 1 & 2 & 3 & 4 & 5 & 6 \\
\hline \# of entities & 4096 & 1536 & 432 & 112 & 27 & 6 & 1
\end{tabular}

An element with a smaller $a$ value is considered to exhibit better quality ( $a$ becomes 1 for a regular tetrahedron).

Cubes without material boundaries. Tetrahedralization patterns of a cube are uniquely determined once the existing information associated with the 18 nodes is provided. Therefore, we skipped all calculations associated with Delaunay triangulation and tetrahedralization (Fig. 7). Although the simplest lookup table will have $2^{18}=262,144$ entries, we reduced the table size to 6,120 entries by using a property of the octree structure (i.e., neighboring cubes exhibit only one level of difference in resolution). Table 2 shows the properties of the generated lookup table. We also developed a specialized reference method that enables fast referencing of this table (see the Appendix for details). The maximum aspect ratio of the tetrahedral elements in this table is 4.5 , which guarantees a high-quality mesh.

\subsection{Performance measurements}

The model-generation times of the previous method [4] and the method presented here are compared in Fig. 8. Here we used a soil-structure-interaction problem with 142 structure components and one soil domain. The elapsed time was compared for a mesh generated at resolutions of $0.4 \mathrm{~m}$ and 0.2 $\mathrm{m}$. Thirty two cores of an SGI UV300, which is a 512 core, 24.5-TB cache-coherent shared-memory system comprising 32 sockets of 16-core Intel Xeon E7-8867 v3 CPUs and 768

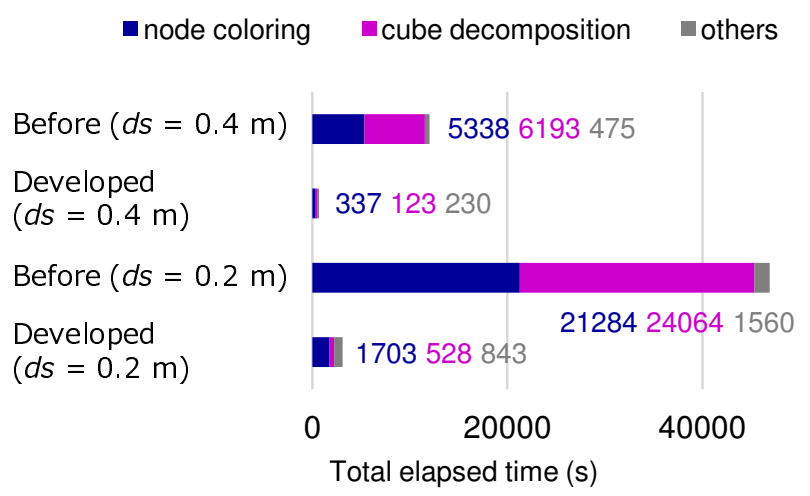

Figure 8: Comparison of mesh-generation times

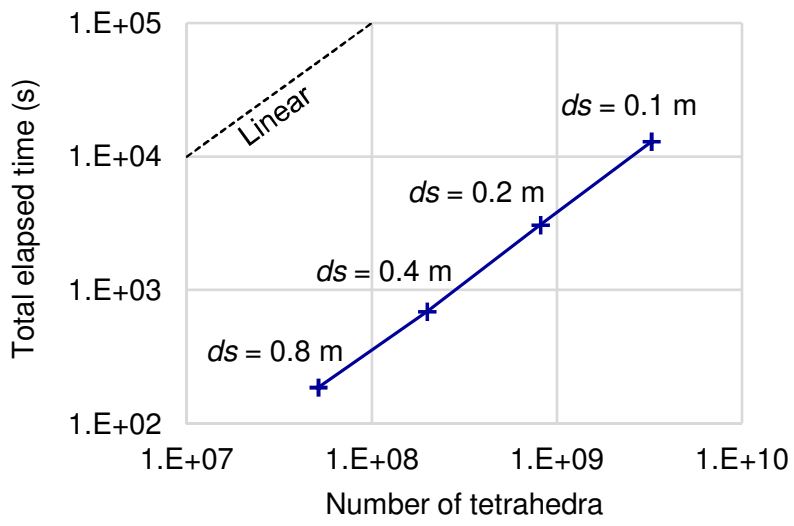

Figure 9: Scalability of the mesh generator presented in this study

slots of 32 GB DDR4 DRAM connected with SGI NUMAlink ASIC technology, were used for meshing. For the 0.4 m-resolution model with 198,675,181 tetrahedral elements, node coloring was accelerated 15.8-fold and tetrahedral mesh generation was accelerated 50.3-fold, thus resulting in a 17.4fold overall increase in the speed. For the $0.2 \mathrm{~m}$-resolution model with 812,815,061 tetrahedral elements, node coloring was accelerated 12.5-fold and tetrahedral mesh generation was accelerated by 45.6 -fold, thus resulting in a 15.3-fold overall increase in speed.

We then compared the elapsed time for different resolutions using the same input model and computational environment. Figure 9 shows that the elapsed time was almost proportional to the number of elements in the generated mesh. Therefore the method proposed in this study was scalable in terms of elapsed time and target-mesh size.

In summary, we developed a fast and robust meshing method applicable to practical wave-propagation simulations with complex geometries and multiple materials. This method was 15-17-fold faster than the previous method [4], and the elapsed time was scalable to the mesh size. In a future work, we will reduce the geometrical approximation by moving, 
adding, and deleting nodes and merging and dividing elements.

\section{FAST DYNAMIC IMPLICIT FINITE-ELEMENT ANALYSIS USING AN ADAPTIVE MULTISTEP PREDICTOR METHOD}

In low-order unstructured finite-element methods, solving Eq. (1) with sparse matrix-vector (SpMV) product-based iterative solvers represents most of the analysis cost. Because this type of computation requires random access and large memory transfers, utilizing the high arithmetic capability of recent computers is difficult. In these dynamic finite-element methods, reducing the number of iterations of the solver and thus reducing the total memory transfer size is effective for increasing the speed of the application. Linear multistep predictors (e.g. the Adams-Bashforth method [7]) are often used for estimating initial solutions and reducing the number of iterations. We can expect further decreases in the number of iterations by improving the accuracy of the predictor. Because the arithmetic capability of recent computers remains idle in finite-element solvers, we developed a highly accurate predictor utilizing this capability [8]. Here we developed an adaptive multistep predictor that reduces the number of iterations and tested its performance on the $\mathrm{K}$ computer using a simple problem set. In this study, we measured the performance of this algorithm on the full system of 2nd generation Intel Xeon Phi-based Oakforest-PACS and the latest Skylake Xeon Gold cluster. We also applied it to a practical problem with complex geometry and multiple materials to further evaluate its performance. In subsequent sections, we summarize the characteristics of the adaptive multistep predictor algorithm [8] and describe the performance-measurement results.

\subsection{Adaptive multistep predictor method}

In this subsection, we generalize the target problem (Eq. (1) and Eq. (2)) and solve $A x_{i}=b_{i}(i=1,2, \ldots)$. In the dynamic finite-element method, the right-hand-side vector $b_{i}$ depends upon the solution of the previous step, $x_{i-1}$. Therefore, the standard solver method involves sequential steps over time (Algorithm 1). Here a standard predictor, such as an AdamsBashforth predictor [7], is used to guess the initial solution. Instead, we developed a predictor that approximately solves several time steps simultaneously in parallel. A previous study by Liu et al. [9] described such an approach. Here $m$ time steps, $(i, i+1, \ldots, i+m-1)$, are solved simultaneously in parallel at step $i$, with these solutions used as initial solutions when solving time steps $i, i+1, \ldots, i+m-1$ (Algorithm 2). Although additional cost is required for the predictor (line 6), a high-performance kernel can be used by the solver, thereby reducing the computational cost of the predictor. In particular, a kernel that computes SpMV products with multiple right-hand-sides (a generalized SpMV (GSpMV)) is used in the predictor solver, which exhibits higher performance levels than a standard SpMV-product kernel, because matrix needs only to be read once from memory, regardless of the number
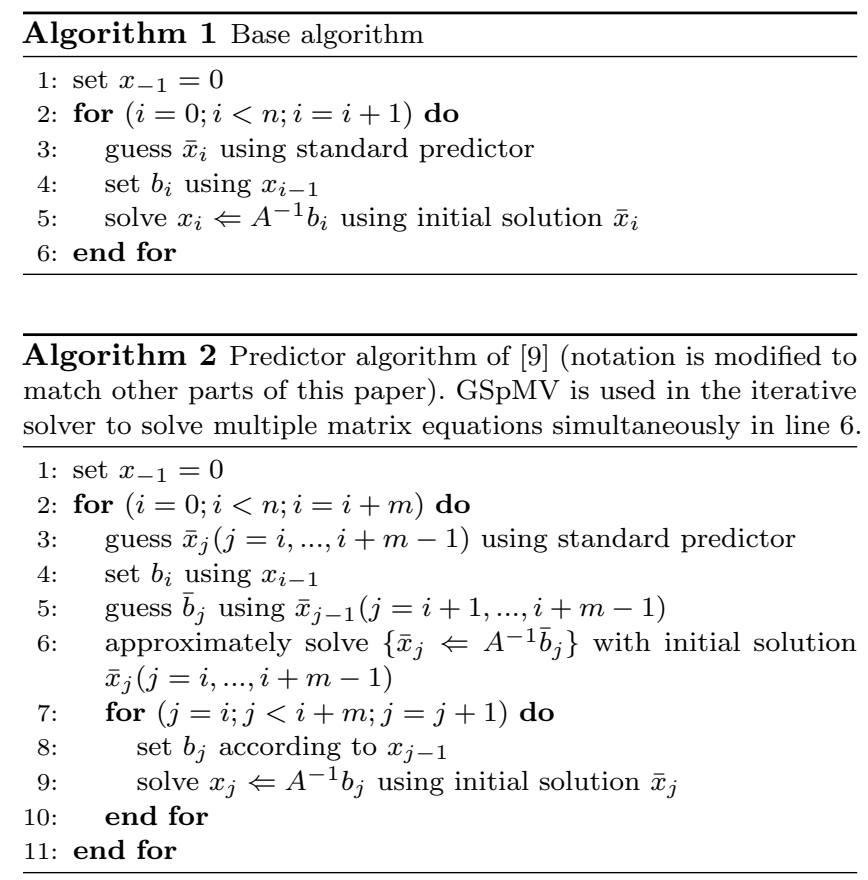

of right-hand sides. Therefore, the reduction in the number of iterations necessary for the solver (line 9) results in speed increases of $30 \%$ for the entire application.

We enhanced this approach to develop a multistep implicit predictor. In the previous method [9], the predicted $\bar{x}$ differs from the actual $x$, because the right-hand-side vectors $\bar{b}_{j}(j=$ $i, \ldots, i+m-1)$ are set based on a standard predictor (line 5 of Algorithm 2). Instead, we adaptively updated $\bar{b}$ based on the partially solved $x$ to improve the predictor accuracy (Algorithm 3). Here line 8 is interrupted and restarted after the right-hand-side vectors $\bar{b}$ are updated on the basis of the partially solved $\bar{x}$ (lines $6-10$ ). In this study, we ran $m$ instances of a conjugate-gradient solver with a $3 \times 3$ block Jacobi preconditioner in line 8 and restarted all of the instances simultaneously at a prescribed iteration threshold. Together with the improvement of the predictor, we expected a higher peak performance because all of the computations were performed by the highly efficient GSpMV kernel.

\subsection{Performance measurements}

4.2.1 Problem settings. We compared the performance of the method with and without the adaptive multistep predictor (Algorithm 1 and Algorithm 3) on a 25 time-step wavepropagation problem in a layered domain. Here four time steps were solved simultaneously in Algorithm $3(m=4)$, and $b$ was updated once in 60 iterations. We implemented both analysis programs based on a conjugate-gradient-based SC15 Gordon Bell Prize finalist solver [10], which is highly tuned for CPU-based computers. Here we modified the matrixvector-product kernels to $3 \times 3$-block compressed row storage (BCRS) format and implemented multiplication for multiple right-hand sides. Because a highly tuned base code was 


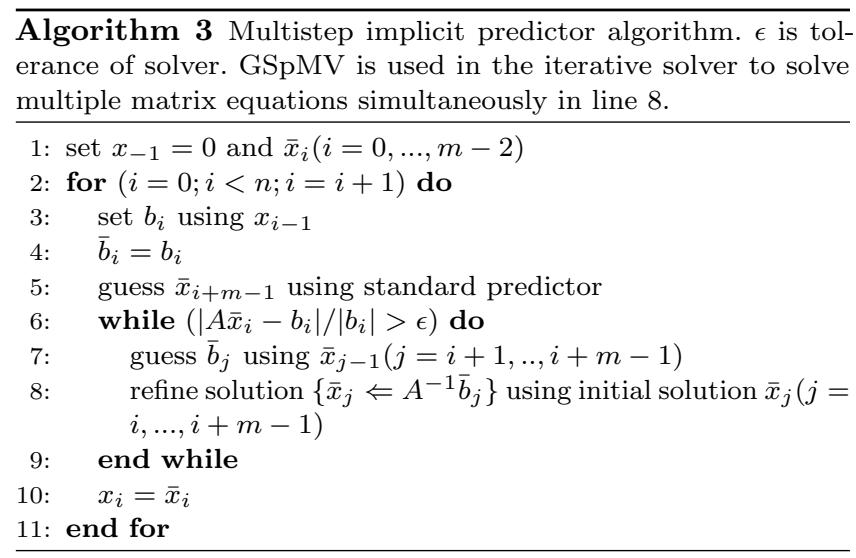

used and the modified parts were highly tuned (i.e., software prefetching was used to hide the indirect access latency of the BCRS matrix-vector kernels), we expected a high performance that reflected the change in the algorithm.

We used Oakforest-PACS, the K computer, a SkylakeSP Xeon Gold cluster, and a Haswell Xeon E5 cluster for performance analyses. Oakforest-PACS [11] is a many-core supercomptuer at the Joint Center for Advanced High Performance Computing (JCAHPC [12]) and comprises 8,208 PRIMERGY CX1640 M1 nodes, each with 68 core Intel Xeon Phi 7250 CPUs connected with the Intel Omni-Path network (100 Gbps). Each node has $16 \mathrm{~GB}$ of MCDRAM (490 GB/s) and $96 \mathrm{~GB}$ of DDR4 memory (115 GB/s). The K computer [13] is a massively parallel supercomputer at the RIKEN Advanced Institute for Computational Science and comprises 82,944 computational nodes, each with eight-core SPARC64 VIIIfx CPUs connected with the Tofu interconnect [14]. Each node has 16 GB of DDR3 memory (64 GB/s). The Skylake-SP Xeon Gold [15] is the latest series of Intel Xeon server CPUs with AVX-512 ISA with up to six channels of DDR4 memory per socket. The Haswell Xeon E5 [16] is a previous version of the Intel Xeon server CPUs with AVX 2.0 ISA with up to four channels of DDR4 memory per socket. Note that the memory bandwidth was markedly increased by the increase in the number of memory channels, as well as memory bus frequency, from the Haswell to the Skylake generation.

4.2.2 Performance on a small scale problem. We first observed the reduction in the number of iterations necessary on a small second-order tetrahedral element model with $5,183,270$ nodes and 3,820,608 elements. The number of iterations reduced by 2.56 -fold from 9,068 to 3,541 by using the predictor described in this study. Because both the SpMV and GSpMV kernels achieved nearly peak memory throughput, GSpMV kernels were computed in 1.2 times the time required for computing a SpMV kernel on the systems tested. This resulted in a 1.42-fold increase in speed on the SkylakeSP Xeon Gold cluster, a 1.40-fold increase in speed on the Haswell Xeon E5 cluster, a 1.69-fold increase in speed on the Oakforest-PACS, and a 1.50-fold increase in speed on the K

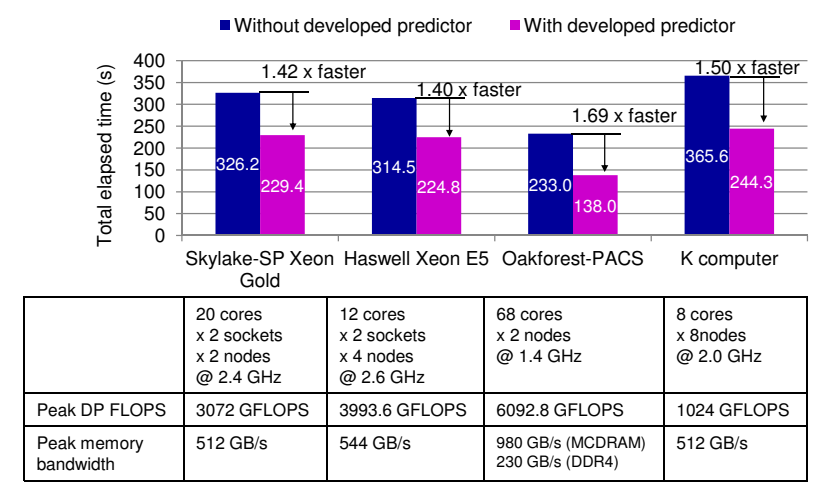

Figure 10: Performance of the method presented in this study. The number of iterations was reduced from 9,068 to 3,541 using the multistep implicit predictor presented here. Note that the MCDRAM bandwidth of Oakforest-PACS is measured STREAM Triad, while DRAM bandwidth are peak memory bandwidth for all of the systems.

computer (Fig. 10). Although it is difficult to directly compare these results given the differences in the target problem, we observed reductions in the number of iterations necessary and speedup greater than or equal to that reported for a previous method [9].

4.2.3 Size-up scalability on the Oakforest-PACS and the K computer. We then assessed the size-up (weak) scalability of the solvers on large computer environments. Because the number of iterations and thus the number of inter-process messages were reduced by the predictor described in this study, we expected improvements in scalability. Table 3 shows the problem sets used for the measurements (WO is for OakforestPACS and WK is for the $\mathrm{K}$ computer). The total number of iterations for Algorithm 1 was almost constant between models and indicated that the model set was suitable for measuring size-up scalability. Figure 11 shows the scalability on the Oakforest-PACS and indicates that the size-up efficiency from 256 nodes to the full system (8,192 nodes) improved from $87.5 \%$ (Algorithm 1) to $91.3 \%$ (Algorithm 3). Figure 12 shows the scalability on the K computer and indicates that the size-up scalability from 1,024 nodes to 32,768 nodes improved from $78.3 \%$ (Algorithm 1) to 80.6\% (Algorithm 3). Moreover, the use of our predictor improved the floating-point-arithmetic efficiency by 2.4 -fold (from $6.0 \%$ to $14.5 \%)$. The time associated with other parts than that of the solver (line 8 of Algorithm 3) was small as compared with the overall runtime, suggesting that the predictor required minimal overhead.

4.2.4 Speed-up scalability on the Oakforest-PACS and the $K$ computer. We expected improvement in speed-up (strong) scalability by the reductions in the number of inter-node messages. Model sets SO and SK (Table 3) represent the model sets used on the Oakforest-PACS and the K computer. 
Table 3: Model configurations. WO and WK are weak scaling model sets for the Oakforest-PACS and the K computer. SO and SK are strong scaling model sets for the Oakforest-PACS and the K computer.

\begin{tabular}{|c|c|c|c|c|c|c|c|}
\hline Model & $\begin{array}{r}\text { \# of computational } \\
\text { nodes }\end{array}$ & $\begin{array}{l}\text { \# of MPI } \\
\text { processes }\end{array}$ & $\begin{array}{r}\text { \# of CPU } \\
\text { cores }\end{array}$ & Degrees of freedom & $\begin{array}{l}\text { Degrees of freedom } \\
\text { per MPI process }\end{array}$ & \# of elements & $\begin{array}{r}\text { Total \# of iterations } \\
\text { for Algorithm } 1\end{array}$ \\
\hline WO-1 & 256 & 1,024 & 16,384 & $1,977,604,941$ & $1,931,255$ & $489,037,824$ & 8,519 \\
\hline WO-2 & 512 & 2,048 & 32,768 & $3,954,085,467$ & $1,930,706$ & $978,075,648$ & 8,460 \\
\hline WO-3 & 1,024 & 4,096 & 65,536 & $7,907,775,462$ & $1,930,609$ & $1,956,151,296$ & 8,401 \\
\hline WO-4 & 2,048 & 8,192 & 131,072 & $15,813,462,837$ & $1,930,354$ & $3,912,302,592$ & 8,318 \\
\hline WO-5 & 4,096 & 16,384 & 262,144 & $31,625,727,090$ & $1,930,281$ & $7,824,605,184$ & 8,318 \\
\hline WO-6 & 8,192 & 32,768 & 524,288 & $63,248,469,975$ & $1,930,190$ & $15,649,210,368$ & 8,268 \\
\hline WK-1 & 1,024 & 1,024 & 8,192 & \multicolumn{4}{|c|}{ same as WO-1 } \\
\hline WK-2 & 2,048 & 2,048 & 16,384 & \multicolumn{4}{|c|}{ same as WO-2 } \\
\hline WK-3 & 4,096 & 4,096 & 32,768 & \multicolumn{4}{|c|}{ same as WO-3 } \\
\hline WK-4 & 8,192 & 8,192 & 65,536 & \multicolumn{4}{|c|}{ same as WO-4 } \\
\hline WK-5 & 16,384 & 16,384 & 131,072 & \multicolumn{4}{|c|}{ same as WO-5 } \\
\hline WK-6 & 32,768 & 32,768 & 262,144 & \multicolumn{4}{|c|}{ same as WO-6 } \\
\hline$\overline{\mathrm{SO}-0}$ & $\overline{128}$ & $\overline{512}$ & $\overline{8,192}$ & $\overline{1,977,604,941}$ & $3,862,510$ & $\overline{489,037,824}$ & - \\
\hline SO-1 (WO-1) & 256 & 1,024 & 16,384 & $1,977,604,941$ & $1,931,255$ & $489,037,824$ & 8,519 \\
\hline $\mathrm{SO}-2$ & 512 & 2,048 & 32,768 & $1,977,604,941$ & 965,627 & $489,037,824$ & 8,533 \\
\hline $\mathrm{SO}-3$ & 1,024 & 4,096 & 65,536 & $1,977,604,941$ & 482,814 & $489,037,824$ & 8,546 \\
\hline $\mathrm{SO}-4$ & 2,048 & 8,192 & 131,072 & $1,977,604,941$ & 241,407 & $489,037,824$ & 8,567 \\
\hline SK-1 (WK-1) & 1,024 & 1,024 & 8,192 & \multicolumn{4}{|c|}{ same as $\mathrm{SO}-1$} \\
\hline SK-2 & 2,048 & 2,048 & 16,384 & \multicolumn{4}{|c|}{ same as $\mathrm{SO}-2$} \\
\hline SK-3 & 4,096 & 4,096 & 32,768 & \multicolumn{4}{|c|}{ same as $\mathrm{SO}-3$} \\
\hline SK-4 & 8,192 & 8,192 & 65,536 & \multicolumn{4}{|c|}{ same as $\mathrm{SO}-4$} \\
\hline SK-5 & 16,384 & 16,384 & 131,072 & $1,977,604,941$ & 120,703 & $489,037,824$ & 8,707 \\
\hline
\end{tabular}

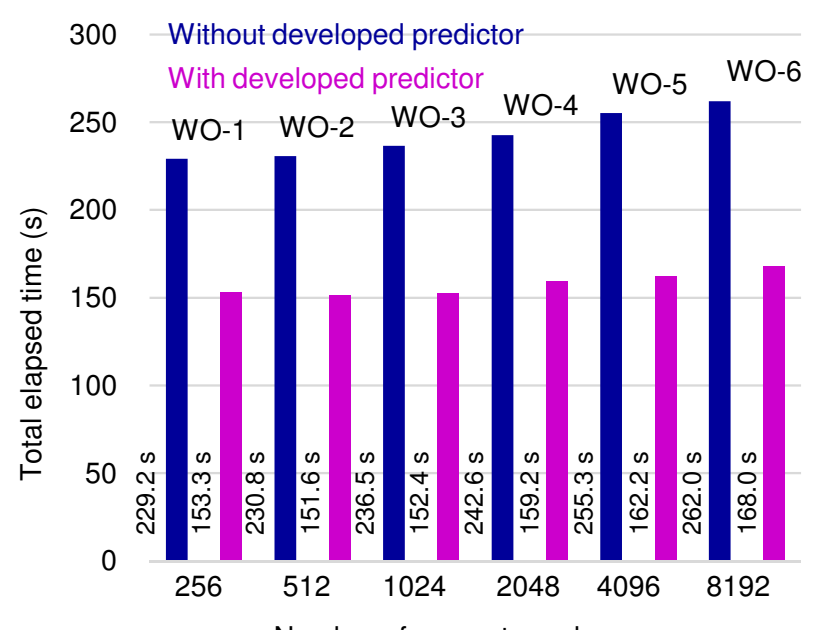

Figure 11: Size-up scalability of solvers with and without the predictor presented in this study on the Oakforest-PACS

Figure 13 (Oakforest-PACS) and Fig. 14 (K computer) show constant increases in speed through the use of our predictor. Because it is more difficult to reduce latency than to increase the throughput of interconnections using the current technology, predictor methods enabling reductions in the number of iterations and interprocess messages will be more effective in the future.

In summary, we developed an adaptive multistep predictor that predicts accurate initial solutions using highly efficient GSpMV kernels, which reduced the number of iterations and memory transfer sizes, and resulted in increases in the speed of the overall application, as well as improved scalability

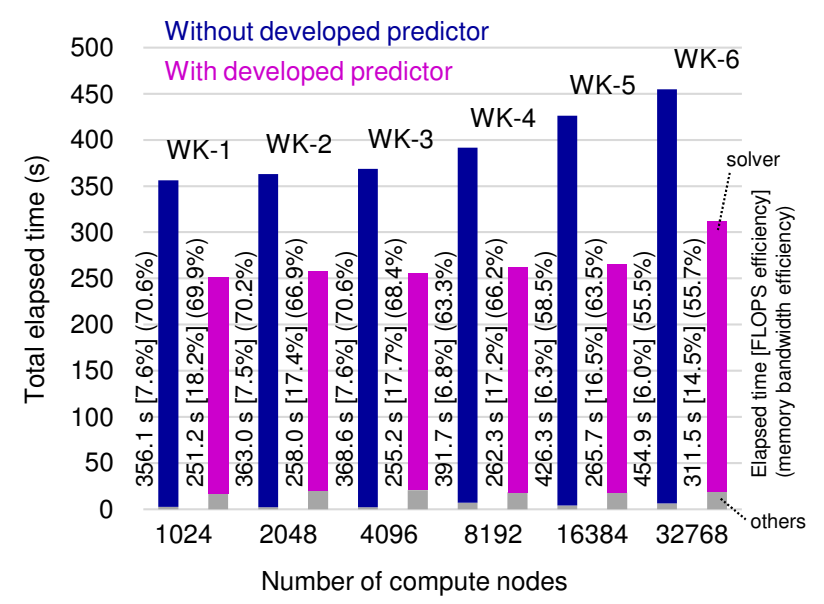

Figure 12: Size-up scalability of solvers with and without the predictor presented in this study on the K computer

on large computer environments. Although we applied our predictor to a simple iterative solver, it can also be used with more sophisticated iterative solvers (e.g., iterative multigrid solvers). Therefore, we expect that our predictor can potentially increase the speed associated with solving a wide range of dynamic finite-element problems.

\section{APPLICATION EXAMPLE}

As an example of wave-propagation analyses in complex shaped multi-material domains, we solved a problem involving earthquake wave-propagation in an underground Tokyo station, in which we were able to obtain high-resolution threedimensional data. Here we targeted a 1,024 $\mathrm{m} \times 1,024 \mathrm{~m} \times$ 


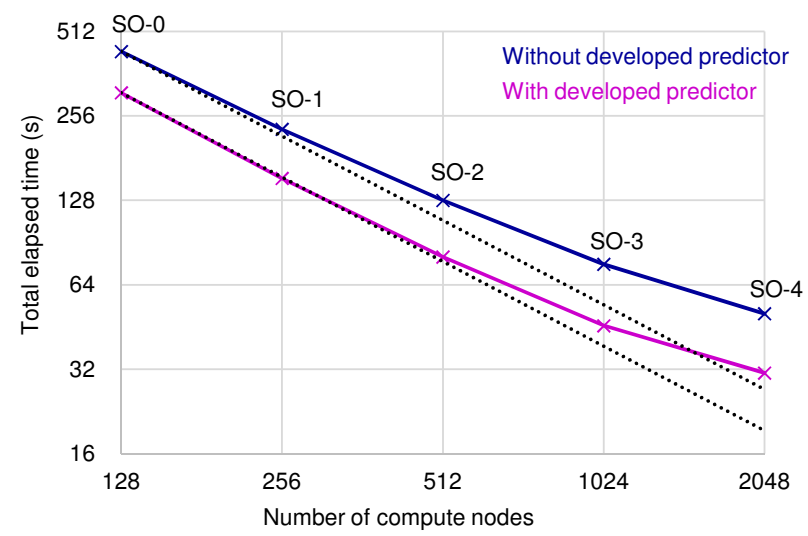

Figure 13: Speed-up scalability of solvers with and without the predictor presented in this study on the Oakforest-PACS

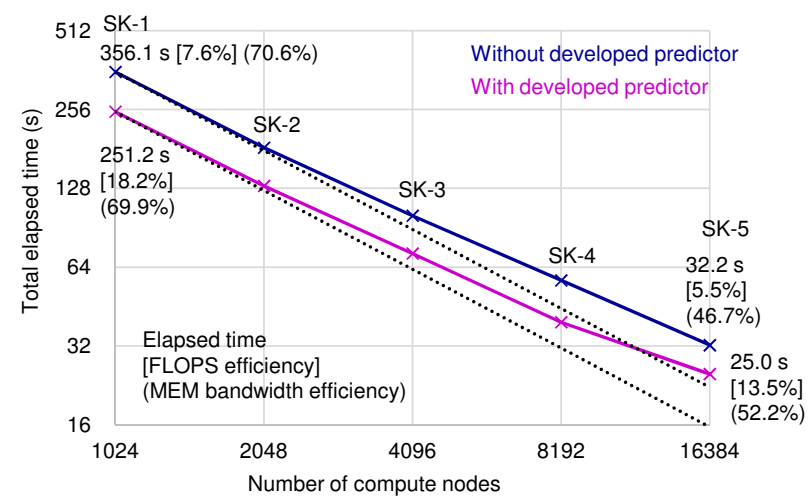

Figure 14: Speed-up scalability of solvers with and without the predictor presented in this study on the K computer

$203 \mathrm{~m}$ area modeled using a three-layered ground structure based on a digital ground map from the Japanese Geotechnical Society [17] and a 5-m grid elevation map [18]. We generated a mesh with $0.25-\mathrm{m}$ sized tetrahedral elements comprising 7,738,790,187 degrees of freedom, 1,869,216,850 elements, and 2,579,596,729 nodes. The model was generated in $5 \mathrm{~h} 26 \mathrm{~min}(19,570 \mathrm{~s})$ using 64 cores of the shared-memory system described in Section 3. Figure 15 describes the finiteelement model, and shows models comprising three-layered ground and underground structures comprising several stories. We input earthquake waves observed during the 1995 Southern Hyogo Prefecture Earthquake (JMA Kobe [19]) into the bottom of the model. The computation of time-step increments of $d t=0.002 \mathrm{~s} \times 7,490$ steps required $4 \mathrm{~h} 23$ min on half of the $\mathrm{K}$ computer system (41,472 computational nodes). The first 25 time steps required $44.1 \mathrm{~s}$ for the conventional solver (Algorithm 1), whereas our method required $29.5 \mathrm{~s}$, providing a 1.50 -fold increase in speed. This result showed that the adaptive multistep predictor enabled speedup for solving actual wave-propagation problems with complex geometries and multiple materials. Figure 15 shows the displacement response at time $t=12.0 \mathrm{~s}$, indicating a complex response according to the underground soil-layer geometry and structure. Such a response can only be analyzed with full three-dimensional wave-propagation analysis. Although we solved a wave-propagation problem associated with an underground structure, this method can be used for medical or other engineering simulations at high resolution.

\section{SUMMARY AND FUTURE PROSPECTS}

Here we described the development of a fast, low-order, finiteelement meshing and analysis method targeting improvements in the quality of wave-propagation simulation results in domains with multiple materials and complex geometries. We accelerated an octree-based, multi-material, unstructured meshing method by fast inner/outer evaluation and lookuptable-based mesh generation, resulting in a 17 -fold increase in speed relative to the base algorithm. We also accelerated a dynamic finite-element solver used on massively parallel computer environments by developing an adaptive multistep predictor that reduced the number of solver iterations and memory transfer, resulting in a 1.69-fold increase in speed on the Oakforest-PACS system. As an example of wavepropagation analysis involving complex multi-material problems, we conducted the analysis of underground structures using the $\mathrm{K}$ computer system. Although we only conducted a single large-scale analysis, the method can be used to conduct multiple simulations of smaller problem sizes. We plan to use these methods for model optimization based on observed data and finite-element wave-propagation analysis results in the future.

\section{ACKNOWLEDGMENTS}

Our results were obtained using the Oakforest-PACS at the Joint Center for Advanced HPC and the K computer at the RIKEN Advanced Institute for Computational Science (proposal numbers: hp160221, hp160160, hp160157, and hp170249). We acknowledge the support from the Japan Society for the Promotion of Science (15K18110, 26249066, 25220908, and 17K14719) and the FOCUS Establishing Supercomputing Center of Excellence. We thank the JCAHPC for the Oakforest-PACS CPU hours for the trial use. We thank the faculty and staff of JCAHPC and the engineers at Fujitsu (Computational Science and Engineering Solution DIV., Technical Computing Solutions Unit) and Intel, particularly Professor Toshihiro Hanawa (The University of Tokyo) and Yoshio Sakaguchi (Fujitsu). We also thank the MPI development and Omni-Path engineering teams at Intel for optimizing the MPI startup time and tuning Omni-Path performance. We thank the Operations and Computer Technologies Division of RIKEN AICS and the High Performance Computing Infrastructure helpdesk for their support in the use of the $\mathrm{K}$ computer. We used the digital ground map from 


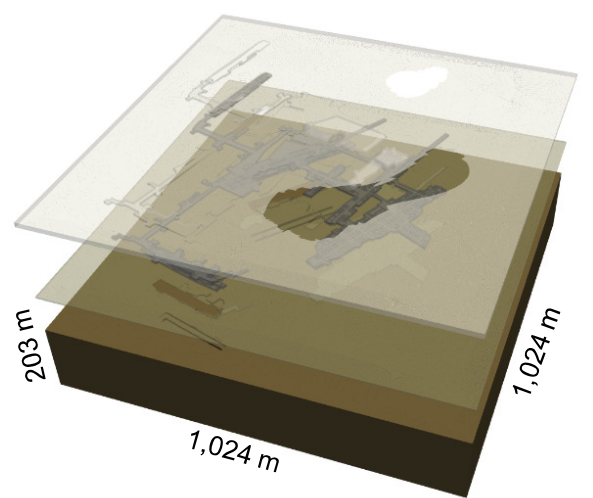

3 layered soil model with subway facility and underground pathway modeled with $0.25 \mathrm{~m}$ sized elements

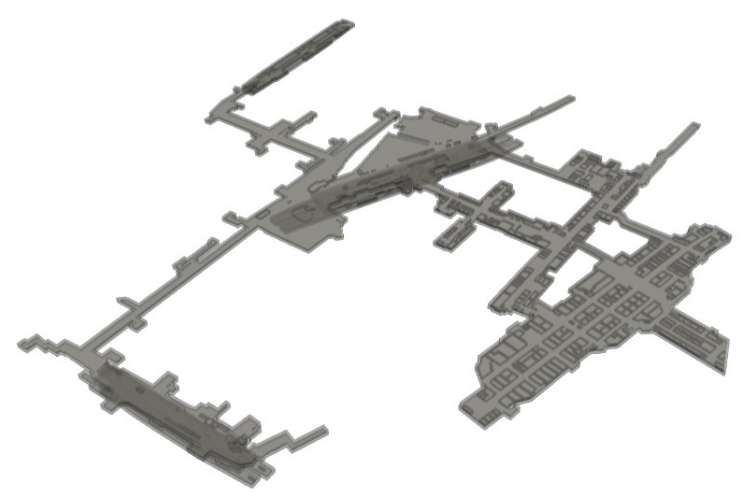

Overview of underground structure

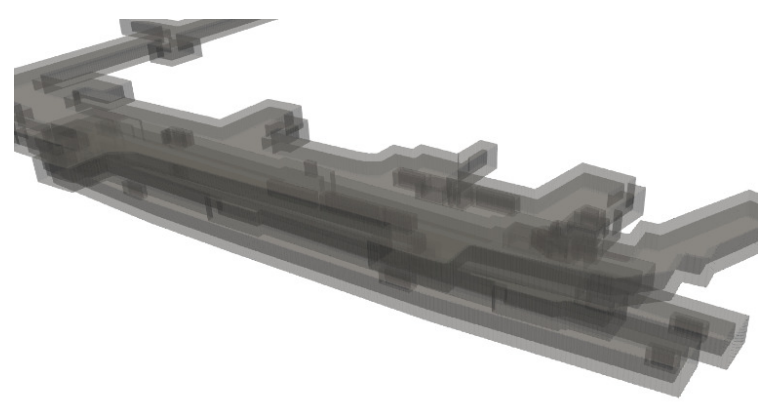

3 story subway facility

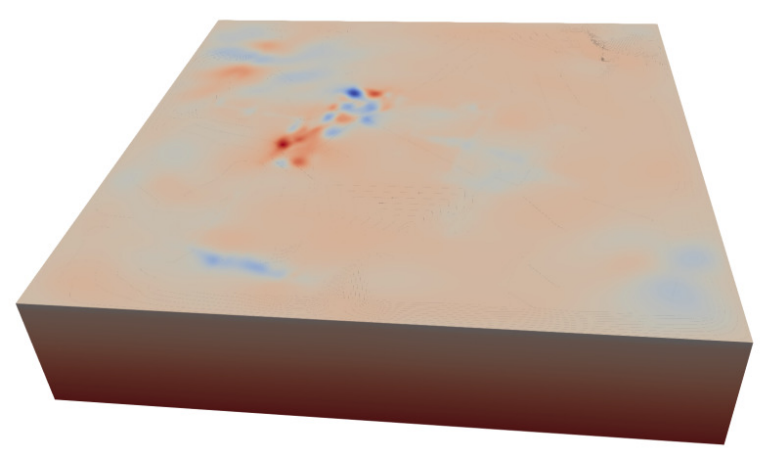

Displacement response at surface

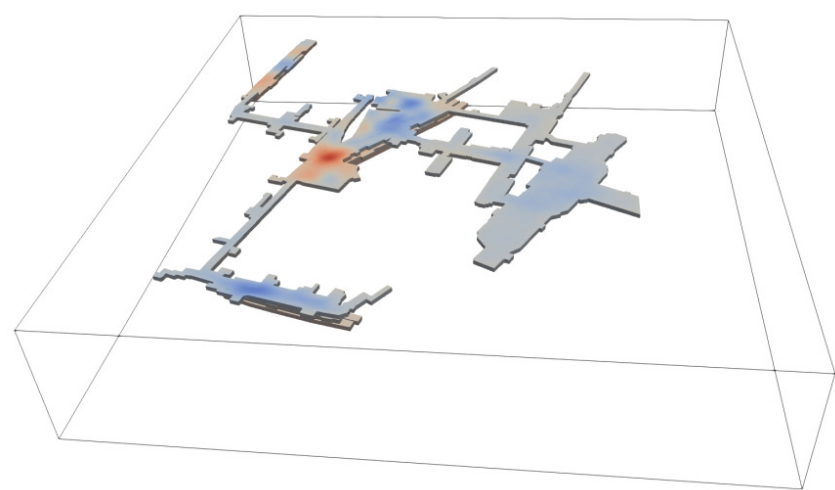

Displacement response of underground structure

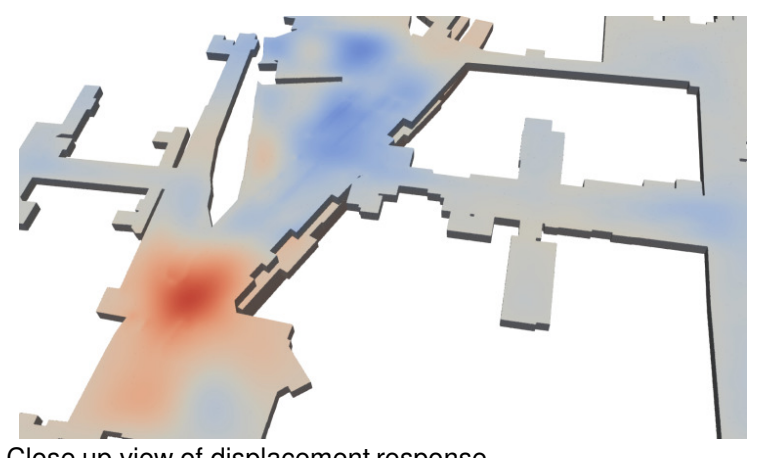

Figure 15: Example wave-propagation analysis performed on a complex-shaped domain with multiple materials. Here, subsurface ground and underground structures of Tokyo station are modeled and analyzed.

the Japanese Geotechnical Society, and a 5-m grid elevation map from the Geospatial Information Authority of Japan.

\section{Appendix}

Here we provide an explanation of the fast-referencing method associated with the lookup tables used for mesh generation.

Cubes with material boundaries. Here we determine the decomposition patterns of a cube from its material identifiers (IDs) at its eight vertices. The key constraint for generating a conforming mesh between neighboring cubes is coping with material-connectivity ambiguities on each face (Fig. 16). In our method, we circumvent this by giving priority to material IDs with smaller magnitudes. Taking this into account, we designed the lookup table referencing algorithm.

First, we counted the number of different material IDs present in the inputs (nmat) and renumbered the material IDs to a sequential local-numbering format from 0 to nmat-1. Here the material ID order is maintained when converting 

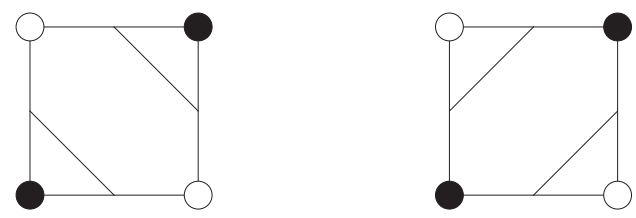

Figure 16: Ambiguity of connectivity on cube faces

from global to local material IDs to enable maintenance of the ambiguity priority. If nmat is two or three, we convert the local material IDs to a decomposition ID in binary or ternary digits. Because all cases of the decomposition patterns are saved in the lookup table in these cases, we can refer to the corresponding row of the lookup table. If nmat is greater than three, we calculate the appearance ID from Table 4 . Here we count the number of duplications of local material IDs. The magnitude of material IDs appearing two or more times can lead to ambiguity; therefore, we check the magnitude and position of these vertices. For each material ID appearing two or more times, we calculate the location ID, which stores the location information of the vertices in nmat decimal numbers. This location ID is a permutation, where the more frequent material IDs and the smaller material IDs are prioritized. However, this location ID cannot contain magnitude information between material IDs on each face. Therefore, if the appearance ID is either 4221, 3221, or 32111, we also calculate the order ID expressing the magnitude of the material IDs used. Using nmat and the IDs described here, the lookup table is searched using a linear search algorithm. The reference procedure is summarized as follows:

(1) Count nmat.

(2) Convert the eight global material IDs to local material IDs.

(3) If nmat is two or three, calculate the decomposition ID and refer to the corresponding row of the lookup table [END]. Otherwise goto 4.

(4) According to nmat, calculate the appearance ID from Table 4.

(5) According to the appearance ID, calculate the location ID.

(6) Calculate the order ID if necessary.

(7) Find a decomposition pattern by linear search of the lookup table corresponding to nmat and the appropriate IDs [END].

As an example, we consider a case where the eight input material IDs are $(7,23,8,7,23,7,15,15)$. In this case, nmat is four (material IDs: 7, 23, 8, and 15). After converting the global material IDs to local material IDs, this pattern becomes $(0,3,1,0,3,0,2,2)$. The appearance ID becomes 3221 because 0 appears three times, and 2 and 3 appear twice. Because the local material ID 0 appears most frequently and the local material ID 2 is smaller than the local material ID 3 and both of them appear twice, the location ID becomes 1089 , 20480 , and 260 , where $4^{0}+4^{3}+4^{5}=1089$ for local material ID 0 , $4^{6}+4^{7}=24080$ for local material ID 3 , and $4^{1}+4^{4}=260$ for local material ID 2. Moreover, the calculated order ID becomes 1, because the most frequent material number, 0 , is the smallest among 0,3 , and 2. Finally, the cube-decomposition pattern is referenced by linear search using these nmat and IDs.

Table 4: Appearance IDs in the boundary cubedecomposition lookup table

\begin{tabular}{cccccc}
\hline \# of materials & 4 & 5 & 6 & 7 & 8 \\
\hline Appearance ID & 5111 & 41111 & 311111 & 2111111 & 11111111 \\
& 4211 & 32111 & 221111 & & \\
& 3311 & 22211 & & & \\
& 3221 & & & & \\
& 2222 & & & & \\
\hline
\end{tabular}

Cubes without material boundaries. Determination of decomposition patterns from the lookup table using the existing information from the 18 nodes requires an 18-bit binary array which is set according to the existence of the 18 nodes (referred to as the decomposition ID). Although it is possible to perform a linear search using this decomposition ID, we save the computational search cost by considering the number of face-centered nodes ( $\mathrm{nf} \mathrm{cn}$ ). By considering $\mathrm{nf} \mathrm{cn}$ as a hash value and only searching the corresponding bucket, we save the computational search cost. Therefore, the reference procedure is summarized as follows:

(1) Count nf cn.

(2) Calculate the decomposition ID.

(3) Find the decomposition pattern by linear search of the lookup table corresponding to $\mathrm{nf} \mathrm{cn}$ and the decomposition ID [END].

\section{REFERENCES}

[1] Pher Errol B. Quinay, Tsuyoshi Ichimura, and Muneo Hori, Waveform Inversion for Modeling Three-Dimensional Crust Structure with Topographic Effects, Bulletin of the Seismological Society of America, 102, pp 1018-1029, 2012 June, doi: 10.1785/0120110175.

[2] T. Ichimura, M. Hori, H. Kuwamoto, "Earthquake Motion Simulation with Multi-Scale Finite Element Analysis on Hybrid Grid," Bulletin of the Seismological Society of America, 97, pp. 1133-1143, 2007.

[3] T. Ichimura, M. Hori, J. Bielak. "A Hybrid multiresolution meshing technique for finite element three-dimensional earthquake ground motion modeling in basins including topography," Geophysical Journal International, 2009, 177, pp. 1221-1232.

[4] K. Fujita, K. Katsushima, T. Ichimura, M. Hori, and M. Lalith. gOctree-based Multiple-material Parallel Unstructured Mesh Generation Method for Seismic Response Analysis of Soil-Structure Systems.h Procedia Computer Science, 80, 2016, pp. 1624-1634.

[5] Ziji Wu and John M. Sullivan. Multiple material marching cubes algorithm. International Journal for Numerical Methods in Engineering, 58(2):189-207, 2003.

[6] Hang Si. Tetgen, a delaunay-based quality tetrahedral mesh generator. ACM Trans. Math. Softw., 41(2):11:1-11:36, February 2015.

[7] Hairer, E., Norsett, S. P., Wanner, G., 1993, Solving ordinary differential equations I: Non stiff problems (2nd ed.), Springer.

[8] K. Fujita, T. Ichimura, M. Horikoshi, M. Hori, and L. Maddegedara. Adaptive multistep predictor for accelerating dynamic implicit finite-element simulations, submitted to the International Conference on High Performance Computing, Networking, Storage and Analysis, (SC'17), 2017.

[9] X. Liu, E. Chow, K. Vaidyanathan, and M. Smelyanskiy. "Improving the Performance of Dynamical Simulations Via Multiple Right-Hand Sides," IEEE 26th International Parallel and Distributed Processing Symposium, 2012.

[10] T. Ichimura, K. Fujita, P.E.B. Quinay, L. Maddegedara, M. Hori, S. Tanaka, Y. Shizawa, H. Kobayashi, and K. Minami, "Implicit nonlinear wave simulation with $1.08 \mathrm{~T}$ DOF and $0.270 \mathrm{~T}$ unstructured 
finite elements to enhance comprehensive earthquake simulation," Proceedings of the International Conference on High Performance Computing, Networking, Storage and Analysis, (SC'15), 2015.

[11] Oakforest-PACS, [Online].

http://www.cc.u-tokyo.ac.jp/system/ofp/index-e.html

[12] JCAHPC, [Online]. http://jcahpc.jp/eng/index.html

[13] H. Miyazaki, Y. Kusano, N. Shinjou, F. Shoji, M. Yokokawa, and T. Watanabe. "Overview of the K computer system," FUJITSU Sci. Tech. J., 48, 3, 302-309, 2012.

[14] Y. Ajima, T. Inoue, S. Hiramoto and T. Shimizu. "Tofu: interconnect for the K computer," FUJITSU Sci. Tech. J., 2012, Vol.48, No.3, pp.280-285

[15] Intel Skylake-SP Xeon Gold series: https://newsroom.intel.com/ newsroom/wp-content/uploads/sites/11/2017/07/intel-xeonscalable-processors-overview.pdf

[16] Intel Xeon E5 v3 series: https://software.intel.com/enus/articles/intel-xeon-processor-e5-2600-v3-product-familytechnical-overview

[17] National Digital Soil Map, The Japanese Geotechincal Society, [Online].

http://www.denshi-jiban.jp/

[18] 5m mesh digital elevation map, Tokyo ward area, Geospatial Information Authority of Japan, [Online]. http://www.gsi.go.jp/MAP/CD-ROM/dem5m/index.htm

[19] Strong ground motion of The Southern Hyogo prefecture earthquake in 1995 observed at Kobe JMA observatory, Japan Meteorological Agency, [Online].

http://www.data.jma.go.jp/svd/eqev/data/kyoshin/jishin/

hyogo_nanbu/dat/H1171931.csv 